\title{
Surgical Outcomes of Minimally Invasive Transforaminal Lumbar Interbody Fusion in Elderly
}

\author{
Jwalant Patel $^{1}$, Vishal Kundnani ${ }^{1}$, Suraj Kuriya ${ }^{2}$, Saijyot Raut ${ }^{1}$, Mohit Meena ${ }^{1}$ \\ ${ }^{1}$ Mumbai Institute of Spine Surgery, Bombay Hospital and Medical Research Centre, Mumbai, ${ }^{2}$ Medical College Baroda, Baroda, \\ Gujarat, India
}

Corresponding Author: Jwalant Patel, MD, MS Spine Fellow, Mumbai Institute of Spine Surgery, Room No-128, Bombay Hospital \& Medical Research Centre, Marine Lines, Mumbai 400020, India

Tel: +91-9979897018

E-mail: jwalant72@gmail.com

Received: December 5, 2019

Revised: December 13, 2019

Accepted: December 13, 2019
Objective: The purpose of this study was to compare the clinical-radiological outcome and incidence of perioperative complications of MIS-TLIF at lower lumbar levels for elderly (Age $>65$ years) and younger patients (Age<65 years). Methods: A retrospective cohort study performed from 2011 to 2017. A total 138 patients who underwent MIS-TLIF were divided into two groups based on age (group $A>65$ years and group $B<65$ years). Perioperative clinical (co-morbidities, surgical time, blood loss, hospital stay, fusion level, VAS, ODI), radiological parameters (fusion, cage subsidence, implant failure), postoperative complications and satisfactory outcomes in the form of Wang's criteria were evaluated in both the groups. A statistical analysis between two matched groups was done with logistic regression analysis, chisquare and student $t$-test. Results: There was no statistical difference in blood loss, surgical time, mobilization and hospital stay between two groups however elderly patients took longer time to become pain free $(p=0.001)$. Both groups showed significant improvement in ODI, VAS and Wang's outcome score however, no statistically significant difference noted in outcome between two groups at final follow up. General complications not affecting outcome were common in elderly group but no statistically significant difference noted among neurological events between both groups. Conclusion: MISS-TLIF surgery in elderly can produce successful clinical outcome and satisfaction after surgery in judiciously selected patients with proper preoperative risk assessment and optimization of medical co-morbidities. Elder age does not prove deterrent to outcome and should not be a contraindication to perform MISSTLIF in lumbar degenerative diseases.

Key Words: Lumbar spine surgery, Elder age, Minimally invasive spine surgery, Decompression, Laminectomy, Fusion, Pedicle screw, Complications, Implant failure, Pseudoarthrosis

\section{INTRODUCTION}

Lumbar degenerative disorders with instability often require spinal stabilization in the form of instrumentation and fusion in elderly patients ${ }^{5,6)}$. Minimally invasive transformainal lumbar interbody fusion (MISS-TLIF) is well established approach that allow access to interbody space at any level of the lumbar spine with minimal retraction of the neural structures ${ }^{4,15)}$. The rationale for such a swing in momentum from open surgery to MISS includes reduced soft tissue damage, reduced blood loss, faster recovery and reduced peri-operative morbidities ${ }^{8,18)}$. With increasing life expectancy, advances in technology and instrumentation in the recent years, the number of MISS-TLIF surgeries for various lumbar pathologies among elderly is on a rise ${ }^{4-6,15)}$. Increasing age has been thought and propagated to produce higher complication rates with increased morbidity and mortality resulting in suboptimal results, which has been substantiated by few authors in literature ${ }^{1,10,11)}$. Majority of literature evaluating efficacy and safety of MISS-TLIF is from studies conducted on younger population (Age $<60$ years) and hence the results of which cannot be extrapolated for elderly population in general ${ }^{19)}$. The effect of age on final outcome and the overall patient satisfaction after MISS-TLIF has been understudied. There are reports of promising results with lumbar fusion surgeries for degenerative disorders in elderly ${ }^{10,11,19)}$. As most of the literature regarding lumbar arthrodesis in elder patients has focused on the prevalence of complications with no comparison to the younger population, the results have not been validated in view of age of the patient.

The primary outcome of this study was to compare the clinical-radiological outcomes including fusion rate, pain free sta- 
tus post surgery and incidence of peri-operative complications of MISS-TLIF at lower lumbar levels for elderly (Age>65 years) and younger patients (Age $<65$ years). The secondary outcome was to evaluate the effect of age on clinical outcome and incidence of complications in two groups.

\section{MATERIALS AND METHOD}

It was a retrospective study of prospectively collected data performed at single institute from 2011-2017. The institutional ethical and review committee approved the study. 176 consecutive patients undergoing MISS-TLIF for degenerative pathology at L4-L5/L5-S1 levels (degenerative lumbar canal stenosis, prolapsed intervertebral disc, spondylolisthesis) with minimum follow up of 24 months were reviewed. A comprehensive clinical and radiological evaluation was conducted on more than one occasion to ascertain the indication of surgery in all patients and only those patients whose symptoms not resolving to dedicated conservative trial with affection of activities of daily life were considered for surgical intervention. A single surgeon at single institute following the same preoperative evaluation and postoperative mobilization protocols for all the patients operated all patients. Patients with surgery other than L4-L5/L5-S1 level, tandem stenosis, revision surgery, non-degenerative pathology like trauma, tumor, infection, lysis and multilevel surgery were excluded from this study. Patients who did not complete minimum 24 months follow up were excluded from the study.

Surgical technique: Patients who underwent MISS-TLIF had screws placed through paramedian incisions approximately 2 $\mathrm{cm}$ long and $3-5 \mathrm{~cm}$ lateral to the midline. The pedicle was cannulated with a jamshidi needle under anteroposterior and lateral fluoroscopic guidance. The needles were exchanged for guidewires. The serial dilators were used to dilate over the guidewires and the pedicles were tapped using a cannulated tap. Screws were placed with corresponding screw extenders and the rod was introduced with a device through a separate proximal stab incision. After placement of locking-cap screws through the screw extenders and application of compression, the screws were torqued and the screw extenders were removed ${ }^{21)}$. Tubular decompression (20/22 mm tubes- METRx system, Medtronics, Memphis, TN, USA) with partial unilateral laminectomy and inferior facetectomy done under microscope. Next steps were discectomy, end plates preparation and titanium cage insertion with locally achieved bone graft followed by closure in layers (Fig. 1).

A total 138 patients satisfying above mentioned inclusion criteria were isolated for analysis. To study the effect of age, patients were divided into two groups. Group A included 72 elderly patients (Age>65 years) and Group B (control group) included 66 younger patients (Age $<65$ years but $>25$ years of age). Preoperative data like demographic (age, sex, body mass index), clinical (VAS, ODI, neurology), medical co-morbidities (diabetes, hypertension, hypercholesterol, IHD, parkinsonism, thyroid disorders, pulmonary dysfunctions, kidney diseases) and radiological data were evaluated and recorded (Table 1). Intraoperative parameters like operative time, blood loss, intraoperative adverse events (dural tears) and hospital stay were recorded and reviewed by an independent observer (Table 1). Postoperative complications were taken into account for all the patients and were divided into four broad categories (Table 3). Most of the patients were mobilized out of bed on first postoperative day and made to walk with walker. Change of dressing was done at 48 hours after surgery and patients were usually discharged within three-four days. All the patients were
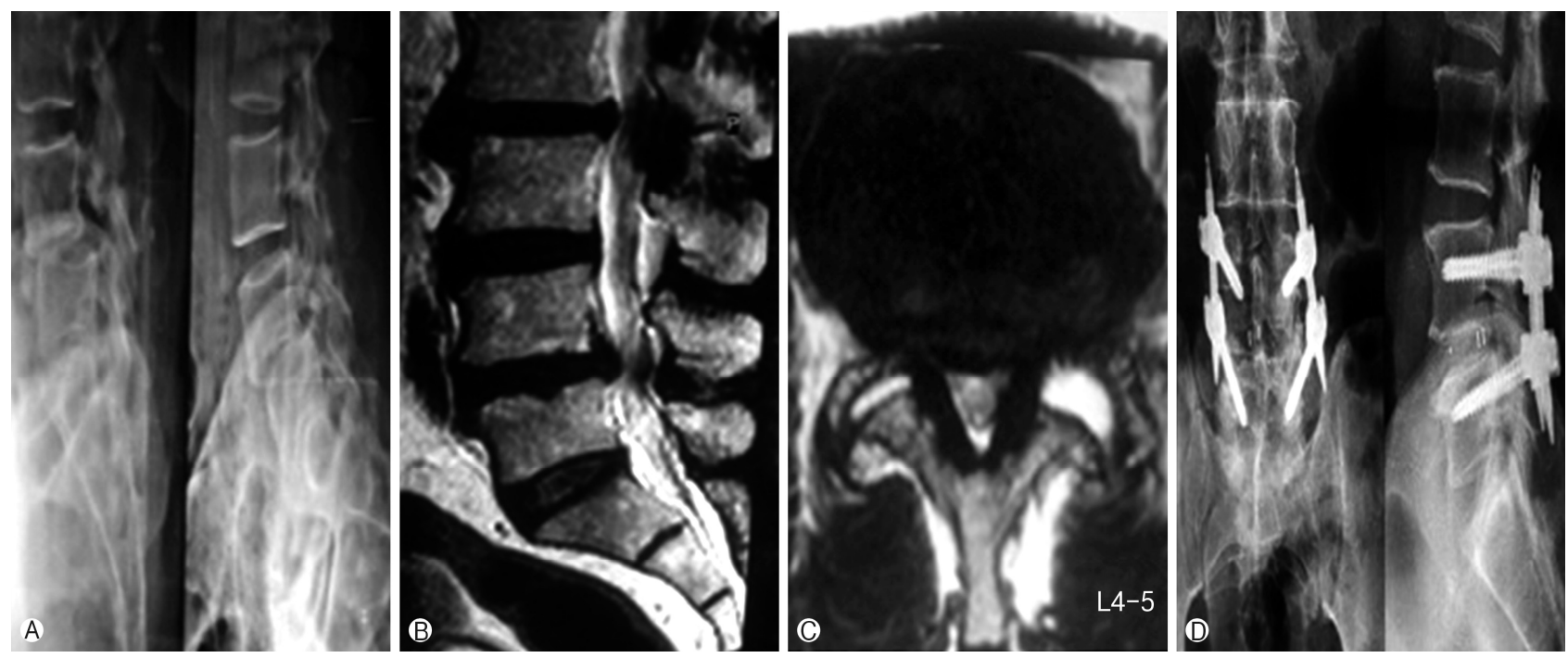

Fig. 1. (A) showing dynamic lumbar spine X-ray with instability and listhesis at L4-L5 level in 75 years old male patient, (B) MRI showing L4-L5 degenerative spondylolisthesis pseudodisc bulge and canal stenosis in sagital T2, (C) showing bilateral facet effusion (left>right) in axial T2 with lumbar canal and lateral recess stenosis, (D) postoperative standing lumbar spine X-ray showing implant with screws and interbody cage in situ at L4-L5 level (MIS-TLIF). 
followed up with evaluation of VAS, ODI and neurological parameters at periodic intervals of 1 month, 3 months, 6 months, 12 months and 24 months. Fusion, screw loosening, implant failure, pseudoarthrosis and cage slippage were assessed in suspected and symptomatic patients with dynamic X-rays and CT scan in both groups at the same follow up protocol periods (Fig. 2) (Table 5). The Wang's criteria were used to evaluate outcome of surgery at final follow up in which grading of results is based on symptom relief, final return to work and satisfaction index (Table 2). All patients were considered to answer a questionnaire and grading of outcome done. Statistical analysis with comparison of two groups was established with simple regression analysis and student $t$-test. The effect of age on clinical outcome and co-relation between presence of medical co-morbidities to complications and clinical outcome was evaluated with chi-square test. P-value $<0.05$ was considered as a significant.

Table 1. Patient demographic and clinical data

\begin{tabular}{|c|c|c|c|c|}
\hline \multicolumn{2}{|l|}{ Parameters } & Elderly group $(\mathrm{N}=72)$ & Younger group $(\mathrm{N}=66)$ & $\mathrm{p}$-value \\
\hline \multicolumn{2}{|l|}{ Age (years) } & $68.8 \pm 4.5$ & $38.6 \pm 12.7$ & - \\
\hline \multirow[t]{3}{*}{ Sex } & Male & 28 & 28 & - \\
\hline & Female & 44 & 38 & - \\
\hline & Male:Female ratio & $0.6: 1$ & $0.7: 1$ & - \\
\hline \multicolumn{2}{|l|}{$\mathrm{BMl}$} & $29.3 \pm 2.5$ & $29.7 \pm 2.8$ & 0.35 \\
\hline \multirow[t]{2}{*}{ Co-morbidities } & Single & 26 & 20 & - \\
\hline & Two or more & 19 & 12 & - \\
\hline \multicolumn{2}{|c|}{ Duration of symptoms (months) } & $9.4 \pm 8.6$ & $9.3 \pm 8.1$ & 0.625 \\
\hline \multirow[t]{2}{*}{ Fusion level } & L4-L5 & 43 & 35 & - \\
\hline & L5-S1 & 29 & 26 & - \\
\hline \multicolumn{2}{|c|}{ Operative time (minutes) } & $185 \pm 35$ & $173 \pm 39$ & 0.058 \\
\hline \multicolumn{2}{|c|}{ Intra-operative blood loss (mL) } & $110 \pm 15$ & $90 \pm 11$ & 0.001 \\
\hline \multicolumn{2}{|c|}{ Hospital stay (days) } & $3.5 \pm 1.57$ & $3.3 \pm 1.76$ & 0.346 \\
\hline \multicolumn{2}{|c|}{ Follow up (months) } & $35.1 \pm 8.8$ & $36.3 \pm 8.7$ & 0.422 \\
\hline
\end{tabular}

BMl: body mass index.
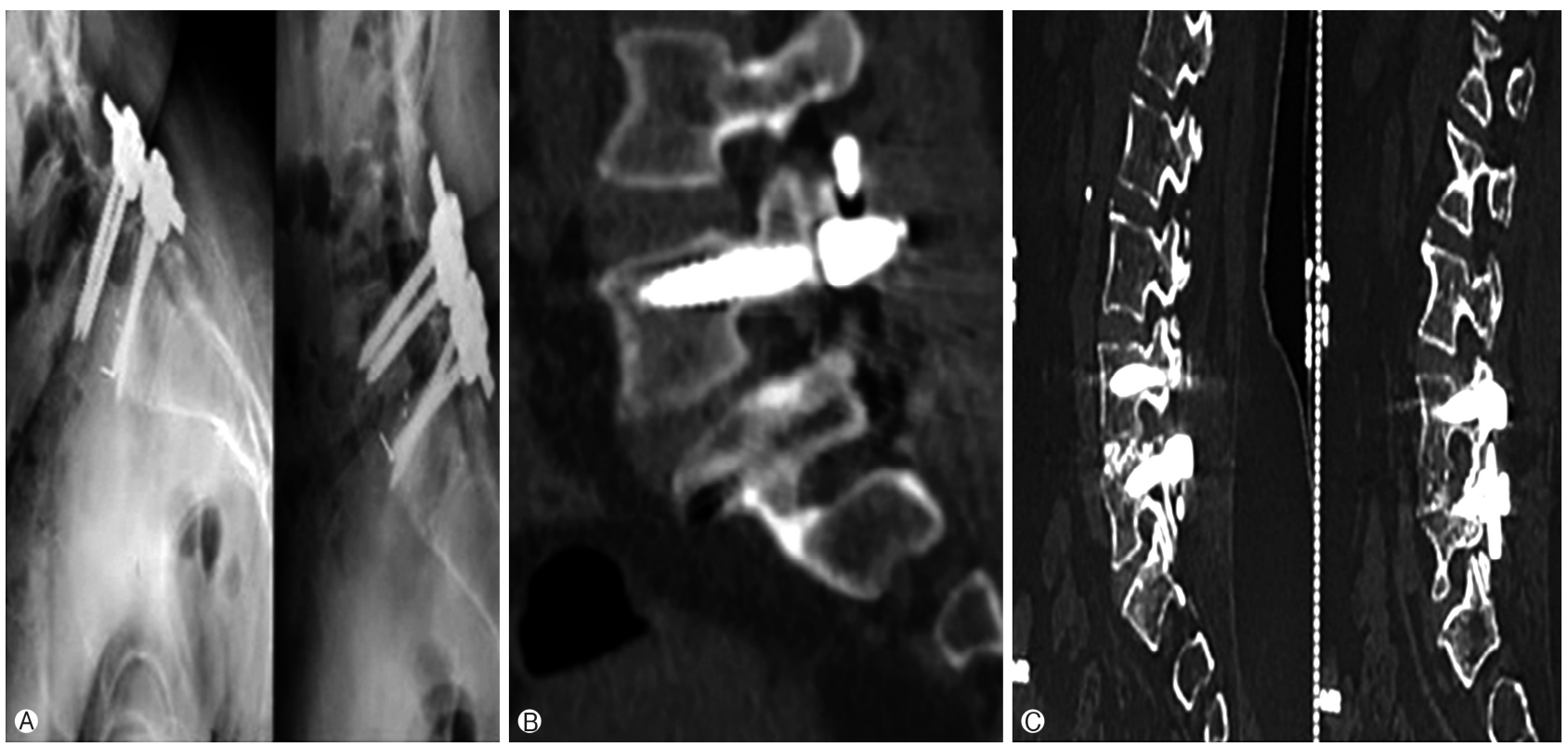

Fig. 2. (A) and (B) postoperative lumbar spine $X$-rays showing progressive migration of cage with screw loosening, implant failure and pseudoarthrosis in 71 years old female, (C) postoperative lumbar spine CT scan showing rigid bone union at L4-L5 level in 68 years old male. 
Jwalant Patel, et al.

Table 2. Wang \& Bohlmans criteria

\begin{tabular}{|c|c|c|c|c|}
\hline Outcome & Pain & Medication & Activity & Work status \\
\hline Excellent & None except for occasional back pain & None & Normal & Normal \\
\hline Good & Markedly improved, occasional pain & $\begin{array}{l}\text { Occasional use of pain } \\
\text { medication }\end{array}$ & $\begin{array}{l}\text { Minimal functional } \\
\text { limitations }\end{array}$ & $\begin{array}{l}\text { Return to work, although } \\
\text { not at the same job activity }\end{array}$ \\
\hline Fair & Some improvement & $\begin{array}{l}\text { Frequent use of pain } \\
\text { medication }\end{array}$ & Restricted & Limited \\
\hline Poor & $\begin{array}{l}\text { No change in symptoms or a worsening } \\
\text { of the patient's condition }\end{array}$ & Oral use of narcotics & Incapacitated & Disabled \\
\hline
\end{tabular}

Table 3. Clinical outcome at final follow up

\begin{tabular}{|c|c|c|c|c|}
\hline \multicolumn{2}{|l|}{ Outcome } & Elderly Group (N=72) & Younger Group (N=66) & p-value \\
\hline \multicolumn{2}{|l|}{ Pre-op VAS } & $8.1 \pm 1.52$ & $7.9 \pm 1.13$ & 0.385 \\
\hline \multicolumn{2}{|l|}{ Post-op VAS } & $3.13 \pm 0.31$ & $3.01 \pm 1.18$ & 0.406 \\
\hline \multicolumn{2}{|l|}{ Pre-op ODI } & $77.45 \pm 6.56$ & $73.13 \pm 6.32$ & 0.001 \\
\hline \multicolumn{2}{|l|}{ Post-op ODI } & $19.15 \pm 5.46$ & $18.89 \pm 6.12$ & 0.792 \\
\hline \multicolumn{2}{|c|}{ Pain free status post surgery (weeks) } & $3.5 \pm 1.3$ & $2.8 \pm 1.3$ & 0.001 \\
\hline Wang \& Bohlmans criteria & $\begin{array}{l}\text { Excellent/Good } \\
\text { Fair/Poor }\end{array}$ & $\begin{array}{l}85.4 \% \\
14.6 \% \\
\end{array}$ & $\begin{array}{l}89.1 \% \\
10.9 \% \\
\end{array}$ & - \\
\hline
\end{tabular}

VAS: visual analog scale; ODI: oswestry disability index.

\section{RESULTS}

A total of 138 out of 176 consecutively operated patients were available till final follow up and were included for statistical analysis. Group A comprised of 72 patients with 28 males and 44 females, whereas Group B comprised of 66 patients with 28 males and 38 females. The mean age for group A was $68.8 \pm 4.5$ years and for group B was $38.6 \pm 12.7$ years. There was no significant difference in body mass index (BMI) between both the groups (Table 1). All patients in-group A had neurogenic claudication as the presenting symptoms with mean duration of symptoms for $9.4 \pm 8.6$ before surgery. In addition to neurogenic symptoms, 66 patients also complained of mechanical low back pain with radiologically evident instability of varying degrees. There was no difference in preoperative mean VAS score ingroup A and in-group B however; there was significant difference in preoperative mean ODI scores between both groups (Table 3). The mean duration of pre-operative symptoms was 9.3 \pm 8.1 months in-group B (Table 1). Medical co-morbidities were found to be present more in-group A with 26 patients with single and 19 cases were with two or more morbidities. In-group B, 20 patients were with single and 12 patients were with two or more co-morbidities (Table 1). The mean operative time was $173 \pm 39$ minutes in-group $B$ and was $185 \pm 35$ minutes in-group $A(p=0.058)$. The mean operative blood loss was $110 \pm 15 \mathrm{~mL}$ in-group $A$ and was $90 \pm 11 \mathrm{~mL}$ in-group $B$ that showed significant difference $(p=0.001)$ between the two groups (Table 1). A single case of minor dural tear was found in both groups that were managed with fibrin glue, local fat graft and watertight fascia closure with no postoperative dural tear related complications. No other intra-operative complication was noticed in both groups. In-group A, 15 patients had complications in postoperative period (Table 4). Four patients (5.5\%) had superficial infection (due to uncontrolled diabetes) and delayed wound healing necessitating prolonged hospital stay and managed conservatively with antibiotics and daily dressings. Two patients $(2.7 \%)$ had deep infection requiring debridement on seventh postoperative day and another patient developed transfusion related problems after multiple blood transfusions on third postoperative period turned into ARDS. One patient with previous history of myocardial infarction developed unstable angina and managed with cardiac team in ICCU. Additionally four patients (5.5\%) had UTI (urinary tract infection) related fever and was managed with oral antibiotics postoperatively without additional hospital stay (Table 4). The mean duration of hospital stay in-group A was 3.5 \pm 1.57 days (Table 1). Four patients had persistent paresthesia related to diabetic neuropathy and none of the patients had any neurological complications in-group $A$ in postoperative period. Seven patients developed SIADH due to electrolyte imbalance and eight patients needed prolonged catheterization (more than 3 days) in-group A. Six patients in-group B had postoperative complications. Two patients (3.03\%) had superficial wound infection managed with daily dressing and another had deep infection requiring debridement and resuturing on seventh post-op day follow up. One patient had malpositioning of screw with persistent paresthesia requiring revision of screw on third postoperative day and two patients had UTI with fever that required prolonged oral antibiotics (Table 4). The mean duration of hospital stay in-group $B$ was $2.45 \pm 1.76$ days. The mean ODI and VAS scores showed 
Table 4. Postoperative complications

\begin{tabular}{|c|c|c|c|}
\hline Category & Complications & Elderly group $(\mathrm{N}=72)$ & Younger group $(\mathrm{N}=66)$ \\
\hline \multirow[t]{4}{*}{ General } & Superficial infection & $4(5.5 \%)$ & $2(3.03 \%)$ \\
\hline & Deep infection & $2(2.7 \%)$ & $1(1.51 \%)$ \\
\hline & Wound dehiscence & 0 & 0 \\
\hline & Fever & $4(5.5 \%)$ & $2(3.03 \%)$ \\
\hline \multirow[t]{5}{*}{ Cardiopulmonary } & Pneumonia & 0 & 0 \\
\hline & ARDS & 1 & 0 \\
\hline & Volume overload (transfusion related) & 0 & 0 \\
\hline & $\mathrm{IHD}$ & 1 & 0 \\
\hline & DVT & 0 & 0 \\
\hline \multirow[t]{2}{*}{ Neurological } & Neurological deficit & 0 & 0 \\
\hline & Paresthesia & $4(5.5 \%)$ & $1(1.51 \%)$ \\
\hline \multirow[t]{3}{*}{ Urinary } & UTI & $4(5.5 \%)$ & $2(3.03 \%)$ \\
\hline & Prolonged catheterization & $8(11.11 \%)$ & $2(3.03 \%)$ \\
\hline & SIADH & $7(9.72 \%)$ & 0 \\
\hline \multirow[t]{5}{*}{ Reoperation } & Screw loosening & $3(4.16 \%)$ & 0 \\
\hline & Pseudoarthrosis & $3(4.16 \%)$ & $1(1.51 \%)$ \\
\hline & ASD & $1(1.38 \%)$ & $2(3.03 \%)$ \\
\hline & Implant failure & $2(2.7 \%)$ & 0 \\
\hline & Cage slippage & $2(2.7 \%)$ & 0 \\
\hline
\end{tabular}

ARDS: acute respiratory distress syndrome; IHD: ischemic heart disease; UTI: urinary tract infection; SIADH: syndrome of inappropriate anti-diuretic hormone secretion; ASD: adjacent segment disease.

significant improvement $(p<0.05)$ in both the groups at final follow up with considerable improvement in clinical parameters (Table 3). There was no statistically significant difference between two groups at final follow up $(p<0.1)$.

As per Wang's Criteria, 61 patients in group A had satisfactory outcomes (excellent to good), 11 had fair to poor outcomes with two patients having only partial relief in symptoms and one patient had worsening of symptoms after surgery. In-group B, 58 patients had satisfactory outcomes (excellent to good) with eight patients having fair to poor outcomes with persistent or recurrent symptoms at final follow up (Table 3). The effect of age on clinical outcome was studied with multivariate analysis that proved that increasing age or addition of fusion does not hamper outcome in elderly population. There was no significant difference in mean time to return to mobilization among two groups although group A took little longer (average 3.4 weeks) to obtain pain-free status compared to group B (average 2.6 weeks). Radiological data analysis showed $90.2 \%$ fusion rate, three cases of screw loosening (Fig. 2), three pseudoarthrosis, two cases each of implant failure (due to trivial fall and low bone mineral density) and cage migration with one case of asymptomatic adjacent segment disease (ASD) in group A (Table 5). Seven patients including two cases of implant failure, three cases of pseudoarthrosis and two cases of cage slippage underwent revision surgeries at the same level while a case of asymptomatic ASD was managed conservatively (Fig. 2). The fusion rate was $93.9 \%$ in-group B with one case each of pseudoarthrosis
Table 5. Radiological outcome at final follow up

\begin{tabular}{lcc}
\hline \hline Outcome & $\begin{array}{c}\text { Elderly Group } \\
(\mathrm{N}=72)\end{array}$ & $\begin{array}{c}\text { Younger Group } \\
(\mathrm{N}=66)\end{array}$ \\
\hline Fusion rate & $65 / 72(90.2 \%)$ & $62 / 66(93.9 \%)$ \\
Screw loosening & 3 & 1 \\
Implant failure & 2 & 0 \\
Pseudoarthrosis & 3 & 1 \\
Cage slippage & 2 & 0 \\
ASD & 1 & 2 \\
\hline
\end{tabular}

ASD: adjacent segment disease.

and screw loosening and two cases of symptomatic ASD at final follow up (Table 5). Revision surgeries were done in three patients- one case of pseudoarthrosis and two cases of symptomatic ASD in-group B (Table 4).

\section{DISCUSSION}

The recent advances in surgical techniques, anesthesiology, patient care and instrumentation design have made MISS-TLIF a safer technique with improved outcomes and decreased morbidity rates ${ }^{13,19,22)}$. This technology has allowed more complex procedures to be performed in populations considered to be at an increased surgical risk (elderly and those with significant co-morbidities) ${ }^{3,9,17)}$. Despite these advancements, age remains 
a major concern when planning a surgical strategy for elderly patients. Although MISS-TLIF for degenerative lumbar disease is being commonly performed nowadays in elderly patients ${ }^{13,22)}$ the evaluation of the outcomes for TLIF in elderly patients has not been widely studied into and there is limited literature related to its comparison with the younger patients ${ }^{17)}$. It is generally said that fusion procedures in elderly are associated with increased cost and incidence of complications ${ }^{3,20)}$. In a recent study, Carreon et al. ${ }^{3)}$ studied 98 patients (age>65 years) who underwent lumbar decompression and fusion. They concluded that elderly patients were at increased risk of surgery related complications. Conversely some authors have reported no difference in either outcomes or rate of complications between the elderly and the younger population ${ }^{12,14)}$. However, the studies that can currently be found point out that there is a clinical benefit for elder patients operated on for degenerative lumbar disease in terms of quality of life ${ }^{2,16)}$. The effect of age on lumbar fusion therefore, remains unclear. Glassman et al. ${ }^{9)}$ had done retrospective review of 85 patients over 65 years old who were treated with single level posterolateral arthrodesis L5-S1 and found a mean improvement at two years of surgery of 6.21 on the physical composite scale of SF-36 and 5.71 points on the mental composite scale of the SF-36. The disability improvement net score as measured with the ODI was 16.38 points. They compared these results with a younger control group and in any case, no significant differences were found. Okuda et al. ${ }^{17)}$ found similar results in terms of quality of life in patients above and below age 70. In their cohort, study of 101 patients affected by degenerative spondylolisthesis at L4-L5 treated using the PLIF technique; they used a questionnaire from the Japanese orthopedic association quantifying both the fusion degree and the complications. They found an improvement without significant differences in both the younger and older group of patients. In some previous studies, the authors have concluded that co-morbidities were associated with greater complication and mortality rates following lumbar surgery ${ }^{1,15)}$ however in the present series, there was no surgical mortality and no difference in the incidence of complications regardless of the presence or absence of medical co-morbidities. Despite the increased co-morbidity rate in patients older than 65 years, extensive adjustment revealed the odds of developing a complication were not significantly different if age was independently assessed ${ }^{2}$. These findings suggest that age itself is not an independent risk factor for complications after MISS-TLIF. Although some clinical case studies ${ }^{4-6)}$ indicate a slightly higher risk of perioperative complications in older patients, cohort studies that have included younger control groups have failed to show significant differences ${ }^{16,17)}$. In this series, a young and an elderly cohort both undergoing single-level MISS-TLIF with substantially similar characteristics, including gender, body mass index and indication for surgery. There was no significant difference between the two groups with respect to total complications. The cardiopulmonary complication rate once said to be common in elderly age group was not very common in this study. One patient developed ARDS requiring prolonged ventilator support with intensive care and prolonged hospital stay but this complication was mostly because of transfusion related volume overload caused by multiple blood transfusions. Over all intra-operative blood loss in two groups was not significantly different. Mortality in elderly population undergoing MISS-TLIF has not been studied in detail in previous studies. This study had no intra or postoperative mortality even after two years of surgery. This is mostly attributed to the fact that this study dealt with degenerative patients with ambulation related issues who were generally healthy except reduced reserves due to age and medical co-morbidities. However, with strict preoperative optimization over morbidities and with judicial patient selection, the mortality related to TLIF in elderly can be brought down to zero as in this study. Reoperation rates were almost the same in both the groups in this study. Clinical outcomes in terms of satisfaction after surgery, as well as pain and functional improvement appear to be similar in elderly patients and younger ones. However, the quality of the evidence as it stands is poor and randomized controlled trials or well-controlled prospective cohort studies are needed to more accurately determine the complication risk and efficacy of MISS-TLIF in elderly patients. As of now, age should not be an independent exclusion factor and the decision to proceed with MISS-TLIF should be made on a case-by-case basis.

The main limitations of study are small sample size, retrospective design, matched pair analysis without randomization even though data collected prospectively. Another limitation was that the study was designed with the assumption that age has a large effect on clinical outcome and peri-operative complications while undergoing MISS-TLIF; if age only had a small effect, it would require a larger sample size to increase the power of the study. Finally, this study was limited to patients undergoing single-level MISS-TLIF and so results may not necessarily apply to patients who undergo multilevel MISS-TLIF. In addition, the authors did not evaluate bone mineral density (BMD) for all the patients and so could not determine relationship between clinical outcome and BMD in elderly patients. Finally, a mid-term follow up of two years has been analyzed here. It does not allow us to know whether differences will appear over a longer period of time.

\section{CONCLUSION}

This study shows a substantial clinical benefit to elderly patients (Age $>65$ years) who underwent MISS-TLIF for degenerative lumbar disease and this improvement in the form of clinical and radiological outcome and complication rate were not different to that obtained by younger patients. Preoperative risk assessment, an appropriate surgical approach and postoperative physical therapy are crucial to successful outcomes in elderly. Medical co-morbidities may need long term preoperative optimization to avoid postoperative and intra-operative complications. Increasing age does not prove deterrent to outcome and should not be a contraindication to perform MISS-TLIF for degenerative lumbar disease. 


\section{REFERENCES}

1. Airaksinen O, Herno A, Turunen V, et al: Surgical outcome of 438 patients treated surgically for lumbar spinal stenosis. Spine 22:2278-2282, 1997

2. B Mohamad, B Nicholas, R. Rafael, et al: Impact of age on shortterm outcomes after lumbar fusion: An analysis of 1,395 patients stratified by decade cohorts. Neurosurgery 77:347-354, 2015

3. Carreon LY, Puno RM, Dimar JR II, et al: Perioperative complications of posterior lumbar decompression and arthrodesis in older adults. J Bone Joint Surg Am 85:2089-2092, 2003

4. Ciol MA, Deyo RA, Howell E et al. An assessment of surgery for spinal stenosis: time trends, geographic variations, complications, and reoperations. J Am Geriatric Soc 44:285-290, 1996

5. Deyo RA, Cherkin DC, Loeser JD, et al: Morbidity and mortality in association with operations on the lumbar spine: the influence of age, diagnosis and procedure. J Bone Joint Surg Am 74:536543, 1992

6. Deyo RA, Ciol MA, Cherkin DC, et al: Lumbar spinal fusion: a cohort study of complications, reoperations and resource use in the Medicare population. Spine 18:1463-1470, 1993

7. Deyo RA, Gray DT, Kreuter W, et al: United States trends in lumbar fusion surgery for degenerative conditions. Spine 30(12): 1441-1445, 2005

8. Foley KT, Gupta SK: Percutaneous pedicle screw fixation of the lumbar spine: preliminary clinical results. Journal of Neurosurgery 9:7-12, 2002

9. Glassman SD, Carreon LY, Dimar JR, et al: Clinical outcomes in older patients after posterolateral lumbar fusion. Spine J 7(5): 547-551, 2007

10. Glassman SD, Polly DW, Bono CM, et al: Outcome of lumbar arthrodesis in patients sixty- five years of age or older. J Bone Joint Surg Am 91:783-790, 2009

11. Gunzburg R, Szpalski M: The conservative surgical treatment of lumbar spinal stenosis in the elderly. Eur Spine J 2:176-180, 2003

12. Herron LD, Mangelsdorf C: Lumbar spinal stenosis: results of surgical treatment. J Spinal Disord 4:26-33, 1991

13. J. D. Schwender, L. T. Holly, D. P. Rouben, K. T. Foley: Minimally invasive transforaminal lumbar interbody fusion (TLIF): Technical feasibility and initial results. Journal of Spinal Disorders \& Techniques 18:S1-S6, 2005

14. Jonsson B, Stromqvist B: Lumbar spine surgery in the elderly. Complications and surgical results. Spine 19:1431-1435, 1994

15. Katz JN, Stucki G, Lipson SJ, et al: Predictors of surgical outcome in degenerative lumbar spinal stenosis. Spine 24:22292233, 1999

16. Kilincer C, Steinmetz MP, Sohn MJ, et al: Effects of age on the perioperative characteristics and short-term outcome of posterior lumbar fusion surgery. J Neurosurg Spine 3:34-39, 2005

17. Okuda S, Oda T, Miyauchi A, et al: Surgical outcomes of posterior lumbar interbody fusion in elderly patients. J Bone $\mathrm{Jt}$ Surg Am 88(12):2714-2720, 2006

18. Park Y, Ha JW: Comparison of one-level posterior lumbar interbody fusion performed with a minimally invasive approach or a traditional open approach. Spine 32(5):537-543, 2007

19. Peng CW, Yue WM, Poh SY, et al: Clinical and radiological outcomes of minimally invasive versus open transforaminal lumbar interbody fusion. Spine 34:1385-1389, 2009

20. Rajaee SS, Bae HW, Kanim L, et al: Spinal fusion in the United States: analysis of trends from 1998 to 2008. Spine 37(1):67-76, 2012

21. Sean M. Jones-Quaidoo, Mladen Djurasovic, R. Kirk Owens II, Leah Y. Carreon: Superior articulating facet violation: percutaneous versus open techniques. J Neurosurg Spine 18:593-597, 2013

22. W. S. Rosenberg, P. V. Mummaneni: Transforaminal lumbar interbody fusion: technique, complications, and early results. Neurosurgery 48:3:569-575, 2001 